\title{
Transcription Profile of Potato (Solanum tuberosum L.) Growing In Vitro
}

\author{
Judit Dobránszki ${ }^{1} \cdot$ Norbert Hidvégi $^{1} \cdot$ Andrea Gulyás $^{1} \cdot$ Bianka Tóth $^{2}$ · Jaime A. Teixeira da Silva ${ }^{1,3}$
}

Received: 22 June 2019 / Accepted: 28 April 2020 / Published online: 12 May 2020

(c) The Author(s) 2020

\begin{abstract}
Despite countless papers on plant tissue culture, few have assessed the transcriptional changes that occur in a developing explant from the moment it is cut and plated, and during early stages of development. In this study, the mRNA profile of in vitro potato (Solanum tuberosum L.) plantlets derived from internode stems was assessed via four comparisons during five time intervals ( 0 h, 24 h, 48 h, 1 week, and 4 weeks) using the Kyoto Encyclopedia of Genes and Genomes (KEGG) classification. After screening KEGG metabolic groups, 35 processes were shown to be either up- or down-regulated. The vast majority (28 processes) were related to growth or development. The expression intensity of 40,430 genes was assessed and $158,107,163$, and 142 sequences were either significantly up- or down-regulated at $0 \mathrm{~h}$ vs $24 \mathrm{~h}, 24 \mathrm{~h}$ vs $48 \mathrm{~h}, 48 \mathrm{~h}$ vs 1 week, and 1 week vs 4 weeks, respectively. A total of 10 DEGs coding for transcription factors were significantly down-regulated, including five ethylene-responsive transcription factors, and two probable WRKY transcription factors. RT-qPCR was used to validate RNA-seq data: Spearman and Pearson correlation coefficients between SeqMonk LFC and RT-qPCR LFC were 0.748 and 0.879 , respectively. To the best of our knowledge, this is the first transcriptomic assessment of the changes taking place in the mRNA profile of a developing potato stem explant.
\end{abstract}

Keywords DEG $\cdot$ Enzyme $\cdot$ Plant growth $\cdot$ Stress $\cdot$ Wounding

\section{Introduction}

A search on any major database for studies related to in vitro plant culture will reveal thousands of papers that describe the response of explants to different growth conditions. What is not so common are studies that have assessed the molecular or transcriptional changes that occur in a developing

Electronic supplementary material The online version of this article (https://doi.org/10.1007/s00344-020-10133-0) contains supplementary material, which is available to authorized users.

Judit Dobránszki

dobranszki@freemail.hu

$\triangle$ Jaime A. Teixeira da Silva jaimetex@yahoo.com

1 Research Institute of Nyíregyháza, Institutes for Agricultural Research and Educational Farm (IAREF), University of Debrecen, P.O. Box 12, Nyíregyháza 4400, Hungary

2 Institutes for Agricultural Research and Educational Farm (IAREF), University of Debrecen, Böszörményi u. 138, 4032 Debrecen, Hungary

3 Present Address: Miki-cho Post Office, Ikenobe 3011-2, P. O. Box 7, Kagawa-ken 761-0799, Japan explant from the moment it is cut and plated, through initial stages of development until the end of subculture, even though considerable evidence exists about the genetic switch of cell fate during in vitro organogenesis (Zhao et al. 2008). Che et al. (2006) built a transcriptomic map of the early developmental events in Arabidopsis thaliana during callus, root, or shoot regeneration after exposure to an auxin-rich callus induction phase followed by a cytokinin-rich shoot induction phase. They found that the biological, cellular, and molecular profile changed depending on the organogenic pathway that had been selected. An earlier study by the same group found that hundreds of genes were expressed during early stages of shoot development (Che et al. 2002). Pischke et al. (2006) noted that histidine kinases were over-regulated in habituated callus, i.e., callus that develops independent of cytokinin during successive subcultures, while several genes involved in cytokinin signaling also showed altered expression. The Arabidopsis Branching Enzyme 1 (BE1) gene, which encodes a glycoside hydrolase that is involved in carbohydrate metabolism, determines the fate of organogenesis and somatic embryogenesis in A. thaliana (Wang et al. 2014). The regenerative ability of cells that underlies totipotency and dedifferentiation lies in the regulation of 
RNA metabolism (Ohtani 2015). Morphogenesis is under the control of a number of mechanisms that regulate gene activity, including the activity of transcription factors (TFs) and microRNAs (Zhuravlev and Omelko 2008).

The ability to identify processes that are activated or repressed during different stages of growth and development of an in vitro plant is of basic biological interest, in terms of development, but could also provide valuable clues for specific metabolic engineering that would target specific processes for up- or down-regulation, either to manipulate growth or physiology, or to overexpress desired metabolites. In this study, we wished to understand the metabolic changes taking place in in vitro stem explants of potato (Solanum tuberosum L.) during a four-week interval, which is the time it takes for axillary shoots and roots to develop. Recent advances using this model plant revealed that when a stem explant is cut, 189 genes are up-regulated, including the increased production of products related to defense, stress response, and wound healing (Teixeira da Silva et al. 2019). Although several stress-related genes were up-regulated in response to ultrasound (US), even during a 4-week developmental period following explant plating, resulting plantlets survived, both after US transmitted by air (Dobránszki et al. 2019) and liquid-transmitted US (PE-US; Teixeira da Silva et al. 2020). A better understanding of the transcriptomic profile would also allow for the developmental fate to be better understood (Tang and Tang, 2017) if the same explants were to be placed on different media with different plant growth regulators, thereby inducing different organs.

\section{Materials and Methods}

\section{Plant material and Growth Conditions}

Plant material as well as culture and growth conditions follow those employed by Dobránszki et al. $(2017,2019)$ and Teixeira da Silva et al. (2019, 2020). Briefly, singlenode segments containing a single leaf were excised from 4-week-old in vitro potato plantlets of 'Desirée' and plated for 4 weeks on plant growth regulator-free Murashige and Skoog (1962) medium. Plant material was sampled at $0 \mathrm{~h}$ (explants); $24 \mathrm{~h}, 48 \mathrm{~h}$ (explants with a developing axillary shoot and adventitious roots); 1 week and 4 weeks (developing explants and growing plantlets). Sampling times based on the growing phases were as determined in Dobránszki et al. (2017) although an additional sampling time was inserted at $48 \mathrm{~h}$ after explants were subcultured. By $48 \mathrm{~h}$ after the start of subculture, rooting percentage of explants exceeded 50\%. Samples (explants at 0, 24 and $48 \mathrm{~h}$; leaves, stems, and roots (i.e., plantlets) at 1 week and 4 weeks; Fig. 1) were stored immediately at $-80{ }^{\circ} \mathrm{C}$ until further analysis.

\section{mRNA Isolation, Library Construction, and Sequencing}

Total RNA was purified from the samples as three biological replicates. Total RNA isolation and removal of rRNA were made as described earlier (Dobránszki et al. 2019; Teixeira da Silva et al. 2019, 2020). The 15 mRNA libraries derived from the three biological replicates of five treatments were pooled into five qualified libraries. Deep sequencing (150 bp paired-end reads, expected data volume was $100 \mathrm{M}$ reads/ sample) was conducted as in our earlier studies (Dobránszki et al. 2019; Teixeira da Silva et al. 2019, 2020).

\section{Bioinformatic Evaluation and Functional Annotation of $\mathrm{mRNA}$-seq Dataset}

Bioinformatic processing and analysis of RNA-seq datasets, as well as subsequent functional annotation of significant DEGs were implemented in the same way and using the same databases and software as reported earlier (Dobránszki et al. 2019; Teixeira da Silva et al. 2019, 2020), namely: SolTub 3.0 (https://plants.ensembl.org/Solanum_tuber osum/Info/Index); FastQC v0.11.7 (https://github.com/sandrews/FastQC) for quality check of the FastQ files and to decide the trimming parameters; TrimGalore v0.5.0 (https://github.com/FelixKrueger/TrimGalore); HISAT2 v2.1.0 (Kim et al. 2015); SeqMonk v1.42.0 program (https ://github.com/s-andrews/SeqMonk); NCBI database using BlastX-fast in Blast2GO v5.2 (Götz et al. 2008); Gene3D, SFLD, SuperFamily, Coils, MobiDBLite, CDD, HAMAP, HMMPanther, HMMPfam, FprintScan, BlastProDom, ProfileScan, HMMTigr, PatternScan; KEGG maps (Kanehisa Laboratories; https://www.kegg.jp/kegg/kegg1.html; Kanehisa et al. 2017). Only those DEGs with a $p$ value $\geq 0.05$ for the intensity difference filter in SeqMonk were considered for the statistical analysis.

\section{Validation of DEGs by RT-qPCR}

Total RNA was isolated from five samples as three biological replicates as described earlier (Dobránszki et al. 2019; Teixeira da Silva et al. 2019, 2020). For the RT-qPCR analysis, we selected eight DEGs from the RNA-seq datasets (PGSC0003DMG400003058, PGSC0003DMG40006825, ENSRNA49463513, PGSC0003DMG400010762, PGSC0003DMG400027054, PGSC0003DMG400028022, PGSC0003DMG400026885, PGSC0003DMG400046976) based on the most negative and positive changes in intensity in the SeqMonk logarithmic fold change (LFC) values. Five reference genes (EFl $\alpha$, elongation factor-1alpha; actin; tubulin; GAPDH, glyceraldehyde-3-phosphate 


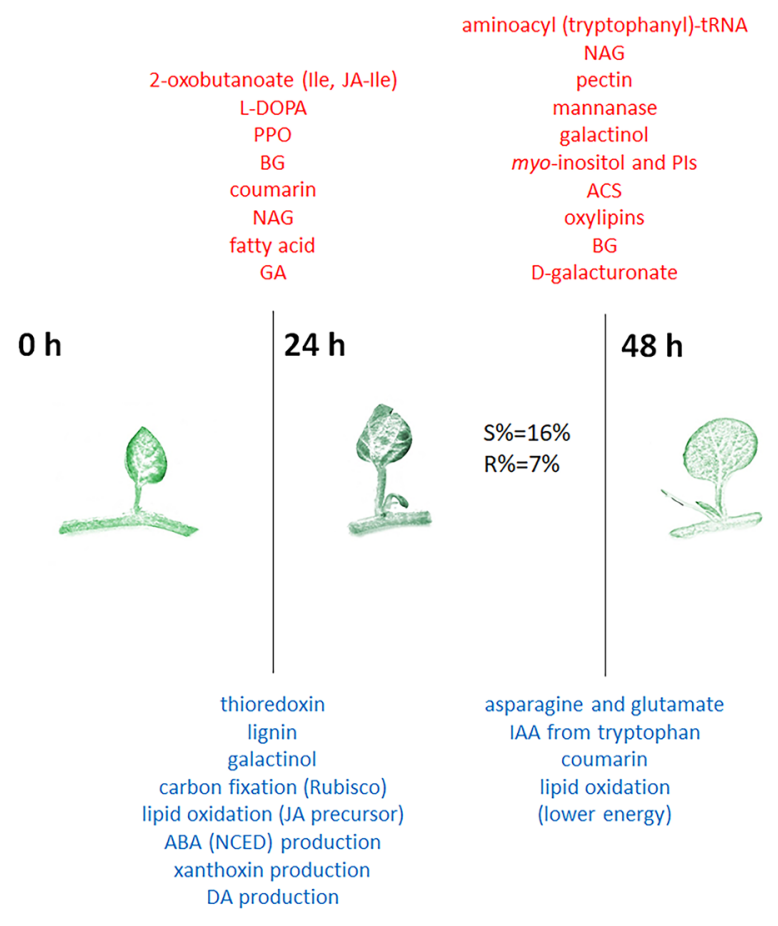

Fig. 1 Hypothetical changes occurring in in vitro single-node stem potato explants over 4 weeks, based on significantly over- or underregulated DEGs. $A B A$ abscisic acid, $A C S$ acetate-CoA synthetase, $B G$ $\beta$-glucosidase, $C K X$ cytokinin dehydrogenase, $D A$ death acid (10-oxo11,15-phytodienoic acid), $F A D$ flavin adenine dinucleotide, $F M N$ flavin mononucleotide, $G A$ gibberellin, IAA indole-3-acetic acid, Ile isoleucine, JA-Ile jasmonoyl-L-isoleucine, L-DOPA L-3,4-dihydroxy-

dehydrogenase; sec 3 , exocyst complex component sec3) were selected for RT-qPCR validation based on a study by Tang et al. (2017) (Teixeira da Silva et al. 2020). RT-qPCR primers (Suppl. Table 4) were developed for the chosen DEGs and the five normalizing (reference) genes ( GAPDH, Actin, sec3, EF1 $\alpha$, tubulin) with the CLC Main Workbench 7.9.2 (Qiagen, Hilden, Germany). geNorm (Vandesompele et al. 2002), NormFinder (Andersen et al. 2004), and BestKeeper (Pfaffl et al. 2004) statistical methods were used to compare the stability of expression intensity among candidate normalizing genes based on the cycle quantification (Cq) value. The results were compared in RefFinder (Pfaffl et al. 2004) based on the geometric mean of the rankings of every single gene calculated by geNorm, NormFinder, and BestKeeper. RT-qPCR was performed and evaluated as in our previous studies (Dobránszki et al. 2019; Teixeira da Silva et al. 2019, 2020). Correlation coefficients (Spearman and Pearson) were calculated using Excel in Microsoft Office 2018 (Microsoft, Redmond, WA, USA).

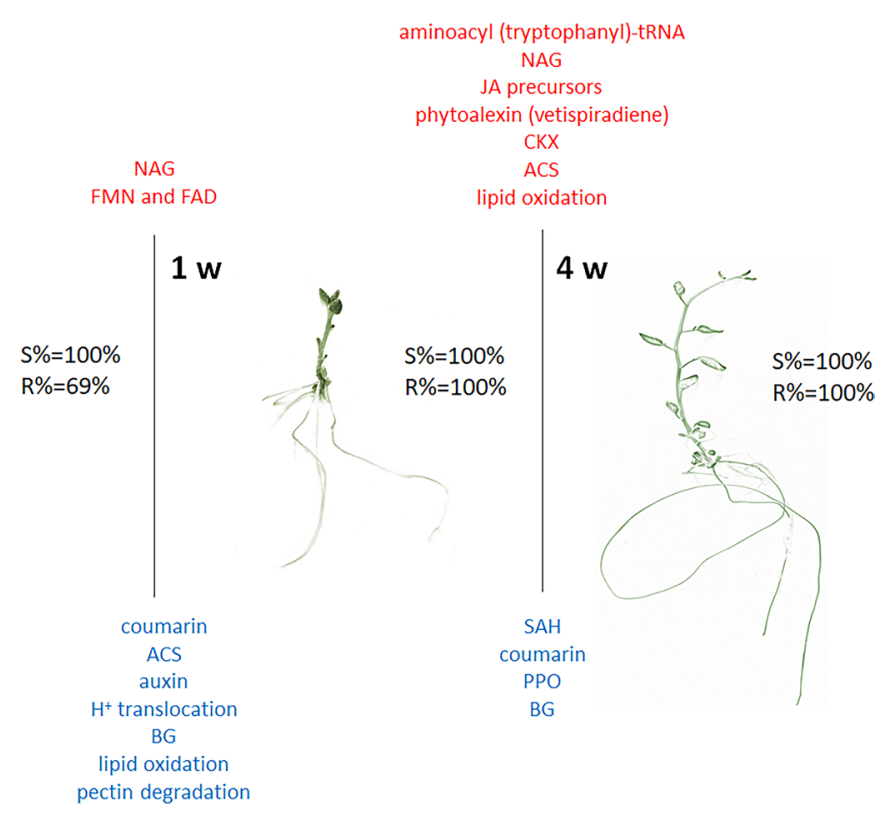

phenylalanine, $N A G N$-acetylglucosamine, $N C E D$ 9-cis-epoxycarotenoid dioxygenase, $P I$ phosphatidylinositol, $P P O$ polyphenol oxidase, $R \%$ rooting percentage (\% of explants forming roots, $n=90$ ), $S \%$, shoot induction percentage ( $\%$ of explants forming shoots; $n=90$ ), $S A H$ S-adenosyl-L-homocysteine. Red=up-regulated functions or other changes; blue $=$ down-regulated functions or other changes

\section{Results}

\section{Global Changes in RNA Expression Profiles}

The expression intensity of 40,430 genes was assessed. A total of $158,107,163$, and 142 sequences were either significantly up- or down-regulated at $0 \mathrm{~h}$ vs $24 \mathrm{~h}, 24 \mathrm{~h}$ vs $48 \mathrm{~h}$, $48 \mathrm{~h}$ vs 1 week, and 1 week vs 4 weeks, respectively (Suppl. Table 1). Except for the $24 \mathrm{~h}$ vs $48 \mathrm{~h}$ comparison, all other comparisons revealed far more down-regulated genes than

Table 1 Number of significantly up- and down-regulated differentially expressed genes (DEGs) (based on GO annotation in Blast2GO) in four comparisons of time intervals during the growth of potato in vitro stem nodal sections

\begin{tabular}{lll}
\hline Treatment comparisons & $\begin{array}{l}\text { \# Up-regulated } \\
\text { DEGs }\end{array}$ & $\begin{array}{l}\text { \# Down- } \\
\text { regulated } \\
\text { DEGs }\end{array}$ \\
\hline 0 h vs 24 h & 36 & 122 \\
24 h vs 48 h & 87 & 20 \\
48 h vs 1 week & 33 & 130 \\
1 week vs 4 weeks & 37 & 105 \\
\hline
\end{tabular}


up-regulated genes (Table 1). Scatter plots show the correlation between any two sets of treatment pairs (Suppl. Fig. 1). Heat maps based on a per-probe normalization (Suppl. Fig. 2) were generated.

\section{RNA-seq/DEG Analysis of Biological, Cellular, and Molecular Processes}

When comparing the significantly up- and down-regulated biological, cellular, and molecular processes (Suppl. Fig. 3; Suppl. Table 2), a cut-off value of $\geq 50 \%$ of DEGs was applied in the comparison across all treatments so as to focus on the most important (weighted) processes. Among the 23 biological processes, the vast majority of significantly upand down-regulated DEGs were limited to six categories (carbohydrate metabolism, catabolism, cellular component organization, cellular protein modification, response to biotic stimulus, and response to stress), the latter process displaying significantly up- and down-regulated DEGs in $100 \%$ of the comparisons. By $24 \mathrm{~h}$ after subculture, six processes were down-regulated while seven processes were upregulated. In the transition between different growth periods, 12 biological processes were down-regulated and seven processes were up-regulated in the transition between 24 and $48 \mathrm{~h}$, while 10 processes each for the transition between $48 \mathrm{~h}$ and 1 week, as well as between 1 and 4 weeks, were up-regulated and 7 processes were down-regulated. Only one significantly up-regulated DEG accounted for a high relative percentage value $(\geq 30 \%)$, namely the response to stress at the transition between 0 and $24 \mathrm{~h}$ (Suppl. Table 2).

Among the 13 cellular processes, the vast majority of significantly up- and down-regulated DEGs were limited to three locations (cell wall, extracellular region, and plastids), the plastids displaying significantly up- and down-regulated DEGs in $50 \%$ of the comparisons. As plants developed in vitro, the number of cellular processes that were up- or down-regulated accounted for less than $50 \%$ of all cellular processes, with the highest number being 5 up-regulated processes between 0 and $24 \mathrm{~h}$ and between 24 and $48 \mathrm{~h}, 6$ down-regulated processes between 24 and $48 \mathrm{~h}$, and 5 downregulated processes between 1 and 4 weeks. When considering a cut-off value of $\geq 35 \%$, six developmental transitions in three locations in in vitro development showed a significantly high proportion of down-regulated DEGs, namely in the extracellular region, membrane, and nucleus, as well as a significantly high proportion of up-regulated DEGs, namely in four developmental transitions in two locations (extracellular region and membrane) (Suppl. Table 2).

Among the 15 molecular processes, the vast majority of significantly up- and down-regulated DEGs were limited to four activities (enzyme regulator activity, hydrolase activity, nucleotide binding, and protein binding), nucleotide binding accounting for $100 \%$ of significantly up- and down-regulated DEGs. Only in the transition between 0 and $24 \mathrm{~h}$ and between 24 and 48 of plant development in vitro did the number of molecular processes that were up- or down-regulated accounted for more than $50 \%$ of all molecular processes, specifically 8 up-regulated processes and 9 down-regulated processes, respectively. When considering the same relative level as biological processes (i.e., $\geq 30 \%$ ), hydrolase activity was significantly up-regulated during in vitro development in the transition between 0 and $24 \mathrm{~h}$ and between 24 and $48 \mathrm{~h}$, but significantly down-regulated in the transition between $48 \mathrm{~h}$ and 1 week. Protein binding was significantly up-regulated during the transition between $48 \mathrm{~h}$ and 1 week, but significantly down-regulated in the transition between 1 and 4 weeks (Suppl. Table 2).

\section{Up- and Down-Regulated DEGs Related to Metabolic Processes and Transcription Factors}

When comparing five sampling times, i.e., four comparisons ( 0 h vs $24 \mathrm{~h}, 24 \mathrm{~h}$ vs $48 \mathrm{~h}, 48 \mathrm{~h}$ vs 1 week, 1 week vs 4 weeks), the focus was only on significantly up- or downregulated DEGs related to growth and development (Table 2; Suppl. Table 3). In amino acid metabolism, DEGs related to nine assumed metabolic processes were either up- or downregulated between $0 \mathrm{~h}$ and 4 weeks (two processes for biosynthesis of other secondary metabolites, eight processes for carbohydrate metabolism, three processes for energy metabolism, five processes for lipid metabolism, four processes for metabolism of terpenoids and polyketides, and two processes for vitamins). DEGs related to aminobenzoate degradation were also up- and down-regulated over the same time interval.

A total of 10 DEGs coding for transcription factors (TFs) were significantly down-regulated, including five ethyleneresponsive TFs, and two probable WRKY TFs (Suppl. Table 5). The vast majority ( $80 \%$ ) were down-regulated between 0 and $24 \mathrm{~h}$, with the remainder (20\%) down-regulated between $48 \mathrm{~h}$ and 1 week.

\section{Validity of DEG Analysis by RT- qPCR}

RT-qPCR was used to validate RNA-seq data. The RefFinder results of the reference genes are shown in Suppl. Table 6. Under in vitro growing conditions, the ranking order (from the most stable to the least stable) was $G A P D H>$ actin $>$ tubulin $>\sec 3>E F 1 \alpha$. We selected the $G A P D H$ gene as the reference gene for DEG validation, as described previously (Teixeira da Silva et al. 2020). According to the RT-qPCR results, all of the chosen DEGs were detected as true-positive up- or down-regulated DEGs (Suppl. Fig. 4; Suppl. Table 6). The correlation coefficients between SeqMonk LFC and RT-qPCR LFC were 0.748 and 0.879 according to Spearman and Pearson correlations, 
Table 2 Number of significantly up- and down-regulated DEGs related to different metabolic processes (based on GO annotation in Blast2GO) in four comparisons of time intervals during the growth of potato in vitro stem nodal sections

\begin{tabular}{|c|c|c|c|c|}
\hline Assumed metabolic group/process & $0 \mathrm{~h}$ vs $24 \mathrm{~h}$ & $24 \mathrm{~h}$ vs $48 \mathrm{~h}$ & $48 \mathrm{~h}$ vs $1 \mathrm{w}$ & 1 week vs 4 weeks \\
\hline \multicolumn{5}{|l|}{ Amino acid metabolism } \\
\hline Alanine, aspartate, and glutamate metabolism & & $1 \downarrow$ & & \\
\hline Aminoacyl tRNA biosynthesis & & $2 \uparrow$ & $2 \downarrow$ & $2 \uparrow$ \\
\hline Cyanoamino acid metabolism & $2 \uparrow$ & $2 \uparrow$ & $2 \downarrow$ & $1 \downarrow$ \\
\hline Cysteine and methionine metabolism & & & & $1 \downarrow$ \\
\hline Glycine, serine, and threonine metabolism & $1 \uparrow$ & & & \\
\hline Selenocompound metabolism & $1 \downarrow$ & & & \\
\hline Tryptophan metabolism & $1 \uparrow$ & $1 \downarrow 1 \uparrow$ & $1 \downarrow$ & \\
\hline Tyrosine metabolism & $1 \uparrow$ & & & $1 \downarrow$ \\
\hline Valine, leucine, and isoleucine biosynthesis & $1 \uparrow$ & & & \\
\hline \multicolumn{5}{|l|}{ Biosynthesis of other secondary metabolites } \\
\hline Flavonoid biosynthesis & $1 \downarrow$ & & & \\
\hline Phenylpropanoid biosynthesis & $1 \downarrow 7 \uparrow$ & $2 \downarrow 4 \uparrow$ & $10 \downarrow 1 \uparrow$ & $9 \downarrow 1 \uparrow$ \\
\hline \multicolumn{5}{|l|}{ Carbohydrate metabolism } \\
\hline Amino sugar and nucleotide sugar metabolism & $2 \uparrow$ & $2 \uparrow$ & $1 \downarrow 7 \uparrow$ & $1 \uparrow$ \\
\hline Fructose and mannose metabolism & & $1 \uparrow$ & & \\
\hline Galactose metabolism & $1 \downarrow$ & $1 \uparrow$ & & \\
\hline Glyoxylate and dicarboxylate metabolism & $1 \downarrow 1 \uparrow$ & & & \\
\hline Inositol phosphate metabolism & & $2 \uparrow$ & & \\
\hline Other glycan degradation & & $1 \uparrow$ & & \\
\hline Pentose and glucuronate interconversions & & $5 \uparrow$ & $2 \downarrow$ & \\
\hline Starch and sucrose metabolism & $2 \uparrow$ & $7 \uparrow$ & $5 \downarrow$ & $3 \downarrow$ \\
\hline \multicolumn{5}{|l|}{ Energy metabolism } \\
\hline Carbon fixation in photosynthetic organisms & $1 \downarrow$ & & & \\
\hline Oxidative phosphorylation & & & $1 \downarrow$ & \\
\hline Phosphatidylinositol signaling system & & $1 \uparrow$ & & \\
\hline \multicolumn{5}{|l|}{ Lipid metabolism } \\
\hline Arachidonic acid metabolism & & $1 \uparrow$ & $1 \downarrow$ & \\
\hline Fatty acid degradation & & $1 \uparrow$ & $1 \downarrow$ & \\
\hline Glycerophospholipid metabolism & $1 \uparrow$ & & & \\
\hline Linoleic acid metabolism & $1 \downarrow$ & & $1 \downarrow$ & $1 \uparrow$ \\
\hline$\alpha$-Linolenic acid metabolism & $1 \downarrow 2 \uparrow$ & & & $1 \uparrow$ \\
\hline \multicolumn{5}{|l|}{ Metabolism of terpenoids and polyketides } \\
\hline Carotenoid biosynthesis & $1 \downarrow$ & & & \\
\hline Diterpenoid biosynthesis & $1 \downarrow$ & & & \\
\hline Sesquiterpenoid and triterpenoid biosynthesis & & & & $1 \uparrow$ \\
\hline Zeatin biosynthesis & & & & $1 \uparrow$ \\
\hline \multicolumn{5}{|l|}{ Nucleotide metabolism } \\
\hline Purine metabolism & & $1 \downarrow$ & $2 \downarrow 1 \uparrow$ & $4 \downarrow$ \\
\hline \multicolumn{5}{|l|}{ Vitamins } \\
\hline Riboflavin metabolism & & & $2 \downarrow$ & \\
\hline Thiamine metabolism & & $1 \downarrow$ & $1 \downarrow 1 \uparrow$ & $4 \downarrow$ \\
\hline \multicolumn{5}{|l|}{ Xenobiotics degradation } \\
\hline Aminobenzoate degradation & $1 \downarrow$ & $2 \downarrow$ & $6 \downarrow$ & $1 \uparrow$ \\
\hline
\end{tabular}

$\uparrow$, up-regulated; $\downarrow$, down-regulated

Details of specific enzymes in Suppl. Table 3 
respectively (Suppl. Fig. 4). These high correlation coefficients indicate a high positive correlation between SeqMonk LFC and RT-qPCR LFC.

\section{Discussion}

This study aimed to assess the changes taking place in freshly prepared stem internode explants that are then grown in medium free of plant growth regulators over 4 weeks, when roots and axillary shoots form. To achieve this, we used KEGG-based classification of metabolic processes based on RNA-seq analysis and DEGs, i.e., a transcriptomic analysis. Transcriptomic profiling of a cut stem explant (Teixeira da Silva et al. 2019) and of an explant that had been exposed to air-based US (Dobránszki et al. 2019) or liquid-based PE-US (Teixeira da Silva et al. 2020) and then left to grow for 4 weeks revealed the up-regulation of several stress-related genes. Plantlets exposed to PE-US showed significantly longer roots, shoot and root fresh weight, and chlorophyll $a / b$ ratio than control plantlets (Teixeira da Silva et al. 2020).

\section{Possible Metabolic Changes Related to Up- and Down-Regulated DEGs Between 0 and $24 \mathrm{~h}$ of the Subculture}

The transcriptomic changes during the first $24 \mathrm{~h}$ of subculture, after placing the explant onto medium, were connected to amino acid, carbohydrate, energy, lipid, terpenoid, and polyketide metabolism and biosynthesis of flavonoids and phenylpropanoids.

Polyphenol oxidases (PPOs) can assist in the oxidation of L-3,4-dihydroxyphenylalanine (L-DOPA) - a non-protein amino acid and inhibitor of electron transport in plants (Mustaq et al. 2013) — to form melanin, producing reactive oxygen species in the process (Soares et al. 2014). While most PPOs are associated with the wounding response, usually in response to abiotic stress, some PPOs are linked with the biosynthesis of metabolites, such as L-DOPA in betalain biosynthesis, tyrosine metabolism in walnut, and formation of 8-8'-linked lignans in creosote bush (Sullivan 2015). The phenylpropanoid pathway has metabolic cross-talk with the biosynthesis of lignins and flavonoids in plants (Li et al. 2010; Mouradov and Spangenberg 2014). The shikimate $O$-hydroxycinnamoyltransferase enzyme, p-coumaroyl-CoA, was down-regulated between 0 and $24 \mathrm{~h}$. In the same period, lactoperoxidase (EC. 1.11.1.7) was similarly up-regulated as was observed immediately after cutting plantlets into explants (Teixeira da Silva et al. 2019). This might increase the production of lignins provided that the precursors are available. The substrate of $\mathrm{p}$-Coumaroyl-CoA has a role in lignin and flavonoid biosynthesis (Vogt, 2010).
When p-coumaroyl-CoA is down-regulated, this results in a decrease in caffeoyl CoA, which is a precursor of S (syringyl) and G (guaiacyl) units of lignin (Hoffmann et al. 2004). In Arabidopsis, reduction in the activity of this enzyme results in reduced lignin production, but the accumulation of flavonoids, and therefore affects the transport of auxin (Besseau et al. 2007; Li et al. 2010). Up-regulated $\beta$-glucosidase at the beginning of subculture ( 0 to $24 \mathrm{~h}$ ) might lead to an increase in coumarin, which is an anti-auxin component in plants (Chattha et al. 2016). In later phases of subculture, $\beta$-glucosidase was down-regulated, which might result in the decreased production of coumarin.

Chitinase was the only enzyme that was down-regulated immediately after explant preparation (i.e., when whole plantlets were cut into explants) causing a decrease in NAG production immediately after cutting (Teixeira da Silva et al. 2019). Thereafter, the up-regulated chitinase, not only between 0 and $24 \mathrm{~h}$, but in all comparisons, i.e., throughout the whole 4-week subculture period, might result in the increased production of $\mathrm{N}$-acetylglucosamine (NAG), which plays a signaling role during plant development (Konopka 2012).

When explants were prepared from whole plantlets, the level of galactinol increased after the up-regulation of DEG for inositol 3- $\alpha$-galactosyltransferase, as described earlier (Teixeira da Silva et al. 2019). However, it was down-regulated between 0 and $24 \mathrm{~h}$, causing decreased galactinol production by $24 \mathrm{~h}$.

Down-regulation of Rubisco might decrease the amount of glycolate, an intermediate of the glycolate pathway (photorespiration) (Allan et al. 2009). Down-regulation of the Rubisco sequence implies not only the modification of glyoxalate and dicarboxylate metabolism, but also the downregulation of carbon fixation (Allan et al. 2009). Catalase, which catalyzes the glycolate glyoxylate conversion, was upregulated at the same time. The $\beta$-glucosidase in cyanoamino acid metabolism is involved in the conversion of $\beta$-D-glucose residues to $\beta$-D-glucose. A closely related homolog sequence for the potato $\beta$-glucosidase and involved in cyanoamino acid metabolism was down-regulated in tomato in response to salt stress (Zhang et al. 2017). The up-regulation of DEGs for $\beta$-glucosidase, glucan endo-1-3- $\beta$-D-glucosidase causes an increase in sugar (glucose and sucrose) production.

Plant oxylipins are oxygenated fatty acids that have a wide range of biological functions in plant growth, development, and protection against stresses (Griffiths 2015). Cytochrome P450, a monooxygenase, catalyzes the metabolism of oxylipins (Griffiths 2015). Lipoxygenases catalyze the oxidation of lipids, which serve as a storage and energy source (Andreou and Feussner 2009). Phospholipase A1 catalyzes the first reaction of the hydrolysis of arachidonic acid (Hanna and Hafez 2018). Increased production of 2-oxobutanoate would result in increased levels of isoleucine and 
jasmonoyl-L-isoleucine (JA-Ile), which is a key hormonal signal (Widemann et al. 2013). Down-regulation of linoleate 13-S-lipoxygenase from 0 to $24 \mathrm{~h}$ caused the decreased production of death acid (10-oxo-11,15-phytodienoic acid, 10-OPDA), which increased in response to the stress caused by explant cutting and preparation at $0 \mathrm{~h}$ (Teixeira da Silva et al. 2019). Similarly, as their precursor, its down-regulation decreased the production of methyl jasmonate and traumatic acid, both of which participate in wound healing.

Threonine ammonia lyase, or threonine deaminase, which is responsible for maintaining the equilibrium of isoleucine in plants together with methionine $\gamma$-lyase (Joshi et al. 2010), was up-regulated in glycine, serine and threonine metabolism, as well as in valine, leucine, and isoleucine biosynthesis, between 0 and $24 \mathrm{~h}$. Thioredoxin reductase regulates photosynthetic enzymes in chloroplasts (Arnér and Holmgren 2000). The reduction of thioredoxin by NADPHdependent thioredoxin reductases may play a part in embryo, root, and shoot apical meristem development (Reichheld et al. 2007; Yano 2014). Catalase, involved in tryptophan metabolism, and which breaks down hydrogen peroxide, for example, in response to abiotic stress (Caverzan et al. 2012), was up-regulated between 0 and $24 \mathrm{~h}$, a similar response that was observed for glyoxylate and dicarboxylate metabolism (carbohydrate metabolism).

The cleavage of 9-cis-epoxycarotenoids to apocarotenoid and xanthoxin, catalyzed by 9-cis-epoxycarotenoid dioxygenase (NCED), is the precursor to the abscisic acid (ABA) biosynthetic pathway (Han et al. 2004). NCED was up-regulated in response to stress caused by explant cutting at $0 \mathrm{~h}$ (Teixeira da Silva et al. 2019). However, $24 \mathrm{~h}$ after placing explants onto regeneration medium, down-regulation of the DEG responsible for NCED caused decreased production of xanthoxin and thus ABA. The A. thaliana NCED3 mutant is unable to accumulate ABA in water-stressed plants, possibly as a result of increased water loss due to impaired stomatal function (Ruggiero et al. 2004), and enhances resistance to multiple abiotic stresses in rice (Huang et al. 2018). Downregulation of NCED results in decreased ABA production. 2-Oxoglutarate-dependent dioxygenase, such as gibberellin $2 \beta$-dioxygenase, is involved in the catabolism and inactivation of gibberellin (Rieu et al. 2008). When working in conjunction with a transcription factor, BRANCHED1, NCED3 suppresses bud development in A. thaliana as a result of the accumulation of ABA (González-Grandío et al. 2016). Down-regulation of gibberellin $2 \beta$-dioxygenase results in increased level of gibberellins.

The above-mentioned changes, which are summarized in Fig. 1, including the accumulation of sugar, modified auxin transport, and an increase in the level of coumarin (an antiauxin agent), an increase in the level of 2-oxobutanoate leading to the increased production of isoleucine and jasmonoylL-isoleucine, an increase in the level of gibberellin, increased production of NAG, fatty acid, L-DOPA but a decrease of thioredoxin and a decrease in the production of methyl jasmonate, traumatic acid, and xanthoxin (and thereby ABA) collectively indicate that explants mitigated the stress caused by explant cutting by $24 \mathrm{~h}$, and initiated the development and growth of new axillary shoots and roots or root meristems from single-node explants. Shoot development was observed in $15 \%$ of explants (vs. root development in $6 \%$ of explants).

\section{Possible Metabolic Changes Related to Up- and Down-Regulated DEGs Between 24 and $48 \mathrm{~h}$ of the Subculture}

Compared to the transcriptomic changes of the first $24 \mathrm{~h}$, between 24 and $48 \mathrm{~h}$, also the metabolisms of vitamins, nucleotide, and xenobiotics were affected but the metabolism of terpenoids and polyketides was not affected.

Asparagine synthetase (AS) catalyzes the formation of asparagine and glutamate (Gaufichon et al. 2016), the former being important for the release of nitrogen for amino acid and protein synthesis (Sieciechowicz et al. 1998). Its downregulation decreases the levels of asparagine and glutamate. Acetate-CoA ligase or acetate-CoA synthetase (ACS) sets the genetic code in the first step of protein synthesis by linking amino acids and tRNA (Pang et al. 2014).

In the indole-3-pyruvate (IPA) pathway, indole-3-acetic acid (IAA), an auxin, can be produced from tryptophan (Zhao 2010). IPA decarboxylase catalyzes the conversion of IPA to indole-3-acetaldehyde (Zhao 2010), which inhibits indole-3-ethanol oxidase (Percival et al. 1973). A. thaliana YUCCA flavoprotein monooxygenase catalyzes a rate-limiting step in the tryptophan-dependent auxin biosynthesis pathway (Zhao 2010).

Not only DEGs for $\beta$-glucosidase, glucan endo-1-3- $\beta$ D-glucosidase, as between 0 and $24 \mathrm{~h}$, but also DEGs for endo-1,4- $\beta$-D-glucanase, sucrose synthase, cellulose synthase (UDP-forming), all of which possibly increase glucose, or sucrose production, and glycogen phosphorylase, which may increase starch degradation, were up-regulated between 24 and $48 \mathrm{~h}$.

Up-regulation of polygalacturonate 4- $\alpha$-galacturonosyltransferase might lead to increased production of pectin (Bar-Peled and O'Neill 2011). Up-regulation of mannan endo-1-4- $\beta$-mannosidase might be connected to the formation of the secondary cell wall (Joët et al. 2014).

The level of galactinol may have increased again by $48 \mathrm{~h}$ if one considers the up-regulation of the DEG responsible for inositol 3- $\alpha$-galactosyltransferase in the $24 \mathrm{~h}$ vs. $48 \mathrm{~h}$ comparison. The raffinose family group of oligosaccharides in plants participates in sugar transport and sugar storage, or they act as signaling molecules, its synthesis starting with the formation of galactinol catalyzed by inositol 3- $\alpha$-galactosyltransferase (Sengupta et al. 2015). 
Up-regulation of PI3P synthase and 1-phosphatidylinositol 4-kinase might lead to increased production of myoinositol and different forms of phosphatidyl-inositols. Inositol phospholipids are important regulators of plant growth (Stevenson et al. 2000), and play a role as signals in intracellular communication and in response to environmental changes (Adepoju et al. 2017). Up-regulation of the 1-phosphatidylinositol 4-kinase sequence might induce changes in energy metabolism and signaling in the plants by overproduction of phosphatidyl-inositol-4-phosphate (PI4P). PI4P has a regulatory role as part of the phosphatidyl-inositol signaling system, otherwise it is the precursor of different phosphatidyl-inositol-biphosphates $\left(\mathrm{PI}(4,5) \mathrm{P}_{2}\right.$ and $\left.\mathrm{PI}(3,4) \mathrm{P}_{2}\right)$ (Stevenson et al. 2000; Williams et al. 2015; Heilmann 2016). Different forms of phosphatidyl-inositol phosphates (mainly 3- and 4-phosphates (PI3P, PI4P)) play specific roles in membrane biogenesis, as well as vesicle and membrane trafficking from the endoplasmic reticulum to the Golgi-apparatus and plasmalemma (Stevenson et al. 2000). $\mathrm{PI}(4,5) \mathrm{P}_{2}$ is a precursor of second messengers (Stevenson et al. 2000). Myo-inositol plays an important role in the biosynthesis of the cell wall, storage of phosphate, inositol and phytic acid, intercellular communication, as part of the phosphatidyl-inositol signaling pathway, and in the storage and transport of auxins (Loewus and Murthy 2000; Williams et al. 2015). Mannanase is linked to the onset of intensive growth from explants by cutting structural mannans in the cell wall, thereby loosening the cell wall during growth (Schröder et al. 2009). Up-regulated pectin demethoxylase and pectin depolymerase might result in the overproduction of D-galacturonate whose pathway can serve as an alternative pathway for the production of ascorbic acid, which is necessary for cell expansion during plant growth, in the Solanaceae (Rigano et al. 2018). Pectate lyase-like genes are necessary for pectin degradation during growth in plants (Leng et al. 2017).

Nucleoside-triphosphate phosphatase was up-regulated at $0 \mathrm{~h}$ in response to explant preparation (cutting) (Teixeira da Silva et al. 2019) but its down-regulation started at $48 \mathrm{~h}$ of tissue culture and increased during subculture causing a change in thiamine production. Down-regulated nucleosidetriphosphate phosphatase in vitamin B1 metabolism (thiamine metabolism) might cause the increased conversion of thiamine diphosphate to thiamine phosphate (Makarchikov 2009). However, sequences coding for adenosine diphosphatase were not affected. Down-regulated acid phosphatase might inhibit the conversion of flavin mononucleotide (FMN) to riboflavin and promote the highest level of FMN and FAD as both are co-factors of several oxidoreductases (Gerdes et al. 2012). However, sequences responsible for FMN hydrolase, which catalyzes the same conversion, were not affected.
Transcriptomic changes detected between 24 and $48 \mathrm{~h}$ (Fig. 1) are mainly related to increased signaling and regulatory activities (increased production of galactinol, myoinositol, phosphatidyl-inositols) pertaining to plant growth and development, increased inter- and intracellular communication (increased production of phosphatidyl-inositols, myo-inositol), and cell wall biosynthesis and secondary cell wall formation (increased activity of mannases, increased production of ascorbic acid and pectin). Increased glucose and sucrose production and starch degradation serve as sources of energy for developmental and growth processes, as was demonstrated for the previous time period (between 0 and $24 \mathrm{~h}$ ). Down-regulation of asparagine-synthase affects both auxin production and asparagine and glutamate levels. Aminoacyl-tRNA biosynthesis and thus protein synthesis increased, partially expressed as increased growth and development. All explants formed axillary shoots, and almost $70 \%$ of these also developed adventitious roots (Fig. 1).

\section{Possible Metabolic Changes Related to Up- and Downregulated DEGs Between $48 \mathrm{~h}$ and 1st Week of the Subculture}

Between $48 \mathrm{~h}$ and 1 week of subculture, during the intensive growth of axillary shoots and adventitious roots when all explants had already formed shoots that had rooted (Fig. 1), only the metabolism of terpenoids and polyketides was not affected out of processes studied and listed in the Table 2 . The metabolism of cyanoamino acid and tryptophan and the biosynthesis of aminoacyl tRNA, which were up-regulated earlier, were down-regulated during this time interval, similarly to the phenylpropanoid biosynthesis, arachidonic acid and linoleic acid metabolisms, fatty acid degradation.

Down-regulated NADH dehydrogenase and $\mathrm{NADH}$ :ubiquinone reductase $\left(\mathrm{H}^{+}\right.$-translocating) might result in a decrease or inhibition of $\mathrm{H}^{+}$translocation and thereby the electrochemical proton gradient through complex I of the electron transport chain in the inner membrane of mitochondria (Hüttemann et al. 2007; Subrahmanian et al. 2016). However, alternative pathways in plants, such as alternative oxidases, or alternative $\mathrm{NAD}(\mathrm{P}) \mathrm{H}$ dehydrogenases, can transport electrons to the electron transport chain by bypassing the route through complex I. The by-passing pathways for electron transfer are dynamically regulated in plants and are based on the plant's metabolic state (Schertl and Braun 2014; Toro and Pinto 2015; Subrahmanian et al. 2016).

Down-regulation of pectin demethoxylase and pectate lyase decreased the degradation of pectin. DEGs responsible for $\beta$-glucosidase, glucan endo-1-3- $\beta$-D-glucosidase, sucrose synthase, and glycogen phosphorylase, which all were upregulated during the first $48 \mathrm{~h}$ of the subculture, were downregulated between $48 \mathrm{~h}$ and 1 week. Down-regulation of glucose-1-phosphate adenylyltransferase results in decrease 
in the production of ADP-glucose and cross-talks to starch and sugar metabolism. Sugar can modify the growth and development of plants including the expansion and division of cells at many levels. For example, it affects the transcription of many genes, maintenance of meristem function, participates in signaling, provides energy, affects biomass, and modulates the level, transport, and/or signal transduction of auxins (Wang and Ruan 2013; Lastdrager et al. 2014; Ivakov et al. 2017), and participates and cooperates in signaling of other hormones like abscisic acid and ethylene (Eveland and Jackson 2012).

\section{Possible Metabolic Changes Related to Up- and Downregulated DEGs Between 1st and 4th Week of the Subculture}

Cysteine and cysteine-related molecules are involved in photosystem protection, as well as in root hair development (Romero et al. 2014). DNA (cytosine-5-)-methyltransferase, involved in cysteine and methionine metabolism mediating the [S-adenosyl-L-methionine + DNA containing cytosine $=$ S-adenosyl-L-homocysteine + DNA containing 5 -methylcytosine] reaction, was down-regulated between 1 and 4 weeks. Up-regulation of linoleate 13-S-lipoxygenase might cause change in the lipid oxidation by the end of the subculture. Vetispiradiene synthase is involved in cyclization during phytoalexin biosynthesis (Bohlmann et al. 1998; Ginzberg et al. 2009) was up-regulated. Cytokinin dehydrogenase, which was up-regulated, catalyzes the irreversible degradation, via oxidation, and thus inactivation of cytokinins (Frébort et al. 2011; Schäfer et al. 2015). As a result of the down-regulation of $\beta$-glucosidase, the production of sugars (glucose, sucrose) decreased, storing energy in macromolecules.

\section{Transcription Factors}

In our study, DEGs for 10 TFs were significantly and mostly down-regulated. Eight of them (ethylene-responsive TF ABR1-like isoform X1, ERF020-like, ERF027-like, ERF061, ERF109-like, probable WRKY TF 40 and 53, and TF bHLH35) were significantly up-regulated immediately after explant preparation $(0 \mathrm{~h})$ in response to cutting (Teixeira da Silva et al. 2019) but down-regulated after explants were cultured on medium for $24 \mathrm{~h}$. Potato explants showed the expression of 21 TF-related DEGs in response to PE-US (Teixeira da Silva et al. 2020). Che et al. (2006) found that an ethylene-responsive factor gene was up-regulated during early adventitious shoot development in in vitro Arabidopsis thaliana.

\section{Conclusion}

A transcriptomic profile now exists for a cut potato stem explant (Teixeira da Silva et al. 2019) and for an explant that had been exposed to US (ultrasound transmitted by air) (Dobránszki et al. 2019) or PE-US (ultrasound transmitted by liquid) (Teixeira da Silva et al. 2020) and then developed for 4 weeks. This study aimed to better understand the transcriptomic changes in a developing single-node stem potato explant during in vitro growth until 4 weeks of age. To achieve this, DEGs pertaining to metabolic processes and their respective theoretical enzymes and/or products were assessed (Fig. 1). We found that $80 \%$ of these processes were clearly linked to plant growth and development as described in detail in the discussion section. Compared to our earlier finding (Teixeira da Silva et al. 2019), we observed changes in DEGs of stress-related enzymes, since the stress caused by explant preparation ceased after explants were cultured for $24 \mathrm{~h}$ on medium. Between 0 and $24 \mathrm{~h}$, when $15 \%$ and $6 \%$ of explants had formed shoots and roots, respectively, production of 2-oxobutanoate, L-DOPA, $\beta$-glucosidase, coumarin, NAG, fatty acid from phospholipids, gibberellin (GA), and the activity of PPO increased, whereas production of lignin, galactinol, Rubisco, lipid oxidation, death acid, and ABA decreased. Between 24 and $48 \mathrm{~h}$, when $100 \%$ and $69 \%$ of explants had formed shoots and roots, respectively, production of NAG, D-galacturonate (ascorbic acid), galactinol, myo-inositol, and different forms of phosphatidyl inositols (PIs), BG, and oxylipins increased, as did growth, and formation of secondary cell walls, whereas production of asparagine, glutamate, IAA, coumarin, and lipid oxidation decreased. Between $48 \mathrm{~h}$ and 1 week (at 1 week, $100 \%$ of explants had also already formed roots), production of NAG, FMN, and FAD increased, whereas production of ACS, coumarin and BG decreased as did the degradation of pectin, auxin biosynthesis, $\mathrm{H}^{+}$translocation, and lipid oxidation. Between 1 and 4 weeks, when several nodes developed with fully developed leaves and an adventitious root system, production of NAG, ACS, JA-precursors, phytoalexin (vetispiradiene) increased, as did aminoacyl (tryptophanyl)tRNA synthesis, degradation of cytokinins, and lipid oxidation, whereas production of coumarin and BG decreased, as did PPO activity.

Acknowledgements Open access funding provided by University of Debrecen (DE). The research was financed by the Higher Education Institutional Excellence Programme (NKFIH-1150-6/2019) of the Ministry of Innovation and Technology in Hungary, within the framework of the Biotechnology thematic programme of the University of Debrecen. The study and submission for publication was approved by the University of Debrecen (BPTR/DEENK/0001/2019).

Data Availability The raw Illumina mRNA-seq data were submitted to NCBI and the processed data were deposited under GEO ID 
GSE123176, BioProject ID PRJNA507769, and SRA ID SRP171630 for the five samples: GSM3498049, GSM3498050, GSM3498051, GSM3498052, GSM3498053.

\section{Compliance with Ethical Standards}

Conflicts of interest The authors declare that they have no conflict of interest.

Open Access This article is licensed under a Creative Commons Attribution 4.0 International License, which permits use, sharing, adaptation, distribution and reproduction in any medium or format, as long as you give appropriate credit to the original author(s) and the source, provide a link to the Creative Commons licence, and indicate if changes were made. The images or other third party material in this article are included in the article's Creative Commons licence, unless indicated otherwise in a credit line to the material. If material is not included in the article's Creative Commons licence and your intended use is not permitted by statutory regulation or exceeds the permitted use, you will need to obtain permission directly from the copyright holder. To view a copy of this licence, visit http://creativecommons.org/licenses/by/4.0/.

\section{References}

Adepoju OA, Williams SP, Gillaspy G (2017) Inositol Phosphates and Energy Signaling in Plants. The FASEB Journal 31(1_ supplement), Biochemistry and Molecular Biology Abstract Number:628.12

Allan WL, Clark SM, Hoover GJ, Shelp BJ (2009) Role of plant glyoxylate reductases during stress: a hypothesis. Biochem J 423:15-22. https://doi.org/10.1042/BJ20090826

Andersen CL, Jensen JL, Ørntoft TF (2004) Normalization of real-time quantitative reverse transcription-PCR data: a model-based variance estimation approach to identify genes suited for normalization, applied to bladder and colon cancer data sets. Cancer Res 64:5245-5250. https://doi.org/10.1158/0008-5472.CAN-04-0496

Andreou A, Feussner I (2009) Lipoxygenases-structure and reaction mechanism. Phytochemistry 70:1504-1510. https://doi. org/10.1016/j.phytochem.2009.05.008

Arnér ESJ, Holmgren A (2000) Physiological functions of thioredoxin and thioredoxin reductase. Eur J Biochem 267:6102-6109. https ://doi.org/10.1046/j.1432-1327.2000.01701.x

Bar-Peled M, O'Neill MA (2011) Plant nucleotide sugar formation, interconversion, and salvage by sugar recycling. Annu Rev Plant Biol 62:127-155. https://doi.org/10.1146/annurev-arplant-04211 $0-103918$

Besseau S, Hoffmann L, Geoffroy P, Lapierre C, Pollet B, Legrand M (2007) Flavonoid accumulation in Arabidopsis repressed in lignin synthesis affects auxin transport and plant growth. Plant Cell 19:148-162. https://doi.org/10.1105/tpc.106.044495

Bohlmann J, Meyer-Gauen G, Croteau R (1998) Plant terpenoid synthases: molecular biology and phylogenetic analysis. Proc Natl Acad Sci USA 95:4126-4133. https://doi.org/10.1073/ pnas.95.8.4126

Caverzan A, Passaia G, Barcellos Rosa S, Werner Ribeiro C, Lazzarotto F, Margis-Pinheiro M (2012) Plant responses to stresses: Role of ascorbate peroxidase in the antioxidant protection. Genet Mol Biol 35(4):1011-1019. https://doi.org/10.1590/S1415-47572 012000600016

Chattha FA, Mehr-un-Nisa, Munawer MA, Kousar S (2016) Coumarin-based heteroaromatics as plant growth regulator. In: Plant
Growth, Chapter 7, pp. 91-105, Intech, Croatia. https://doi. org/10.5772/64854

Che P, Gingerich DJ, Lall S, Howell SH (2002) Global and cytokininrelated gene expression changes during shoot development in Arabidopsis. Plant Cell 14:2771-2785. https://doi.org/10.1105/ tpc.006668

Che P, Lall S, Nettleton D, Howell SH (2006) Gene expression programs during shoot, root, and callus development in Arabidopsis tissue culture. Plant Physiol 141:620-637. https://doi.org/10.1104/ pp. 106.081240

Dobránszki J, Asboth G, Homoki D, Bíró-Molnár P, Teixeira da Silva JA, Remenyik J (2017) Ultrasonication of in vitro potato single node explants: activation and recovery of antioxidant defence system and growth responses. Plant Physiol Biochem 121:153-160. https://doi.org/10.1016/j.plaphy.2017.10.022

Dobránszki J, Hidvégi N, Gulyás A, Teixeira da Silva JA (2019) mRNA transcription profile of potato (Solanum tuberosum $\mathrm{L}$ ) exposed to ultrasound during different stages of in vitro plantlet development. Plant Mol Biol 100(4-5):511-525. https://doi.org/10.1007/s1110 3-019-00876-0

Eveland AL, Jackson DP (2012) Sugars, signalling, and plant development. J Exp Bot 63(9):3367-3377. https://doi.org/10.1093/jxb/ err379

Frébort I, Kowalska M, Hluska T, Frébortová J, Galuszka P (2011) Evolution of cytokinin biosynthesis and degradation. J Exp Bot 62(8):2431-2452. https://doi.org/10.1093/jxb/err004

Gaufichon L, Rothstein SJ, Suzuki A (2016) Asparagine metabolic pathways in Arabidopsis. Plant Cell Physiol 57(4):675-689. https://doi.org/10.1093/pcp/pcv184

Gerdes S, Lerma-Ortiz C, Frelin O, Seaver SMD, Henry CS, de Crécy-Lagard V, Hanson AD (2012) Plant B vitamin pathways and their compartmentation: a guide for the perplexed. J Exp Bot 63(15):5379-5395. https://doi.org/10.1093/jxb/ers208

Ginzberg I, Tokuhisa JG, Veilleux RE (2009) Potato steroidal glycoalkaloids: biosynthesis and genetic manipulation. Potato Res 52:1-15. https://doi.org/10.1007/s11540-008-9103-4

González-Grandío E, Pajoro A, Franco-Zorrillad JM, Tarancón C, Immink RGH, Cubas P (2016) Abscisic acid signaling is controlled by a BRANCHED1/HD-ZIP I cascade in Arabidopsis axillary buds. Proc Natl Acad Sci USA. https://doi.org/10.1073/ pnas. 1613199114

Götz S, García-Gomez JM, Terol J, Williams TD, Nagaraj SH, Nueda MJ, Robles M, Talón M, Dopazo J, Conesa A (2008) Highthroughput functional annotation and data mining with the Blast2GO suite. Nucleic Acids Res 36(10):3420-3435. https:// doi.org/10.1093/nar/gkn176

Griffiths G (2015) Biosynthesis and analysis of plant oxylipins. Free Radic Res 49(5):565-582. https://doi.org/10.3109/10715 762.2014.1000318

Han S-Y, Kitahata N, Sekimata K, Saito T, Kobayashi M, Nakashima K, Yamaguchi-Shinozaki K, Shinozaki K, Yoshida S, Asami T (2004) A novel inhibitor of 9-cis-epoxycarotenoid dioxygenase in abscisic acid biosynthesis in higher plants. Plant Physiol 135:1574-1582. https://doi.org/10.1104/pp.104.039511

Hanna ES, Hafez EAA (2018) Synopsis of arachidonic acid metabolism: a review. J Adv Res 11:23-32. https://doi.org/10.1016/j. jare.2018.03.005

Heilmann I (2016) Phosphoinositide signaling in plant development. Dev 143:2044-2055. https://doi.org/10.1242/dev.136432

Hoffmann L, Besseau S, Geoffroy P, Ritzenthaler C, Meyer D, Lapierre C, Pollet B, Legrand M (2004) Silencing of hydroxycinnamoyl-coenzyme a shikimate/quinate hydroxycinnamoyltransferase affects phenylpropanoid biosynthesis. Plant Cell 16:1446-1465. https://doi.org/10.1105/tpc.020297

Huang Y, Guo Y-M, Liu Y-T, Zhang F, Wang Z-K, Wang H-Y, Wang F, Li D-P, Mao D-D, Luan S, Liang M-Z, Chen L-B (2018) 
9-cis-Epoxycarotenoid dioxygenase 3 regulates plant growth and enhances multi-abiotic stress tolerance in rice. Front Plant Sci 9:162. https://doi.org/10.3389/fpls.2018.00162

Hüttemann M, Lee I, Samavati L, Yu H, Doan JW (2007) Regulation of mitochondrial oxidative phosphorylation through cell signaling. Biochem Biophys Acta 1773:1701-1720. https://doi. org/10.1016/j.bbamcr.2007.10.001

Ivakov A, Flis A, Apelt F, Fünfgeld M, Scherer U, Stitt M, Kragler F, Vissenberg K, Persson S, Suslove D (2017) Cellulose synthesis and cell expansion are regulated by different mechanisms in growing arabidopsis hypocotyls. Plant Cell 29:1305-1315. https://doi. org/10.1105/tpc. 16.00782

Joët T, Laffargue A, Salmona J, Doulbeau S, Descroix F, Bertrand B, Lashermes P, Dussert S (2014) Regulation of galactomannan biosynthesis in coffee seeds. J Exp Bot 65(1):323-337. https:// doi.org/10.1093/jxb/ert380

Joshi V, Joung JG, Fei Z, Jander G (2010) Interdependence of threonine, methionine and isoleucine metabolism in plants: accumulation and transcriptional regulation under abiotic stress. Amino Acids 39(4):933-947. https://doi.org/10.1007/s0072 6-010-0505-7

Kanehisa M, Furumichi M, Tanabe M, Sato Y, Morishima K (2017) KEGG: new perspectives on genomes, pathways, diseases and drugs. Nucleic Acids Res 45:D353-D361. https://doi.org/10.1093/ nar/gkw1092

Kim D, Langmead B, Salzberg SL (2015) HISAT: a fast spliced aligner with low memory requirements. Nat Methods 12(4):357-360. https://doi.org/10.1038/nmeth.3317

Konopka JB (2012) N-Acetylglucosamine functions in cell signaling. Scientifica. https://doi.org/10.6064/2012/489208

Lastdrager J, Hanson J, Smeekens S (2014) Sugar signals and the control of plant growth and development. J Exp Bot 65(3): 799-807. https://doi.org/10.1093/jxb/ert474

Leng Y, Yang Y, Ren D, Huang L, Dai L, Wang Y, Chen L, Tu Z, Gao Y, Li X, Zhu L, Hu J, Zhang G, Gao Z, Guo L, Kong Z, Lin Y, Qian Q, Zeng D (2017) A rice pectate lyase-like gene is required for plant growth and leaf senescence. Plant Physiol 174:11511166. https://doi.org/10.1104/pp.16.01625

Li X, Bonawitz ND, Weng J-K, Chapple C (2010) The growth reduction associated with repressed lignin biosynthesis in Arabidopsis thaliana is independent of flavonoids. Plant Cell 22:1620-1632. https:// doi.org/10.1105/tpc. 110.074161

Loewus FA, Murthy PPN (2000) Myo-Inositol metabolism in plants. Plant Sci 150:1-19. https://doi.org/10.1016/S0168-9452(99)00150 $-8$

Makarchikov AF (2009) Vitamin B1: metabolism and functions. Biochem (Moscow) Suppl Ser B Biomed Chem 3(2):116-128. https://doi. org/10.1134/S1990750809020024

Mouradov A, Spangenberg G (2014) Flavonoids a metabolic network mediating plants adaptation to their real estate. Front Plant Sci 5:620. https://doi.org/10.3389/fpls.2014.00620

Murashige M, Skoog F (1962) A revised medium for rapid growth and bioassay with tobacco tissue culture. Physiol Plant 15:473-497. https://doi.org/10.1111/j.1399-3054.1962.tb08052.x

Ohtani M (2015) Regulation of RNA metabolism is important for in vitro dedifferentiation of plant cells. J Plant Res 128:361-369. https://doi. org/10.1007/s10265-015-0700-4

Pang YLJ, Poruri K, Martinis SA (2014) tRNA synthetase: tRNA aminoacylation and beyond. Wiley Interdiscip Rev: RNA 5(4):461-480. https://doi.org/10.1002/wrna.1224

Pfaffl MW, Tichopad A, Prgomet C, Neuvians TP (2004) Determination of stable housekeeping genes, differentially regulated target genes and sample integrity: BestKeeper-excel-based tool using pair-wise correlations. Biotech Lett 26:509-515. https://doi.org/10.1023/ B:BILE.0000019559.84305.47
Percival FW, Purves WK, Vickery LE (1973) Indole-3-ethanol oxidase. Plant Physiol 51:739-743. https://doi.org/10.1104/pp.51.4.739

Pischke MS, Huttlin EL, Hegeman AD, Sussman MR (2006) A transcriptome-based characterization of habituation in plant tissue culture. Plant Physiol 140:1255-1278. https://doi.org/10.1104/pp.105.07605

Reichheld J-P, Khafif M, Riondet C, Droux M, Bonnard G, Meyera $Y$ (2007) Inactivation of thioredoxin reductases reveals a complex interplay between thioredoxin and glutathione pathways in Arabidopsis development. Plant Cell 19:1851-1865. https://doi. org/10.1105/tpc.107.050849

Rieu I, Eriksson S, Powers SJ, Gong F, Griffiths J, Woolley L, Benlloch R, Nilsson O, Thomas SG, Hedden P, Phillips AL (2008) Genetic analysis reveals that C19-GA 2-oxidation is a major gibberellin inactivation pathway in Arabidopsis. Plant Cell 20:2420-2436. https:// doi.org/10.1105/tpc. 108.0588

Rigano MM, Lionetti V, Raiola A, Bellincampi D, Barone A (2018) Pectic enzymes as potential enhancers of ascorbic acid production through the D-galacturonate pathway in Solanaceae. Plant Sci 266:55-63. https://doi.org/10.1016/j.plantsci.2017.10.013

Romero LC, Aroca MA, Laureano-Marín AM, Moreno I, García I, Gotor C (2014) Cysteine and cysteine-related signaling pathways in Arabidopsis thaliana. Mol Plant 7(2):264-276. https://doi.org/10.1093/ $\mathrm{mp} / \mathrm{sst} 168$

Ruggiero B, Koiwa H, Manabe Y, Quist TM, Inan G, Saccardo F, Joly RJ, Hasegawa PM, Bressan RA, Maggio A (2004) Uncoupling the effects of abscisic acid on plant growth and water relations. Analysis of stol/nced3, an abscisic acid-deficient but salt stress-tolerant mutant in Arabidopsis. Plant Physiol 136:3134-3147. https://doi. org/10.1104/pp.104.046169

Schäfer M, Brütting C, Meza-Canales ID, Großkinsky DK, Vankova R, Baldwin IT, Meldau S (2015) The role of cis-zeatin-type cytokinins in plant growth regulation and mediating responses to environmental interactions. J Exp Bot 66(16):4873-4884. https://doi.org/10.1093/ jxb/erv214

Schertl P, Braun HP (2014) Respiratory electron transfer pathways in plant mitochondria. Front Plant Sci 5:163. https://doi.org/10.3389/ fpls.2014.00163

Schröder R, Atkinson RG, Redgwell RJ (2009) Re-interpreting the role of endo- $\beta$-mannanases as mannan endotransglycosylase/hydrolases in the plant cell wall. Ann Bot 104:197-204. https://doi.org/10.1093/ aob/mcp120

Sengupta S, Mukherjee S, Basak P, Majumder AL (2015) Significance of galactinol and raffinose family oligosaccharide synthesis in plants. Front Plant Sci 6:656. https://doi.org/10.3389/fpls.2015.00656

Sieciechowicz KA, Joy KW, Ireland RJ (1998) The metabolism of asparagine in plants. Phytochemistry 27(3):663-671. https://doi. org/10.1016/0031-9422(88)84071-8

Soares AR, Marchiosi R, Siqueira-Soares RdC, Barbosa de Lima R, Dantas dos Santos W, Ferrarese-Filho O (2014) The role of L-DOPA in plants. Plant Signal Behav 9:e28275. https://doi.org/10.4161/ psb. 28275

Stevenson JM, Perera IY, Heilmann I, Persson S, Boss WF (2000) Inositol signaling and plant growth. Trends Plant Sci 5(6):252-258. https://doi.org/10.1016/S1360-1385(00)01652-6

Subrahmanian N, Remacle C, Hamel PP (2016) Plant mitochondrial Complex I composition and assembly: a review. Biochem Biophys Acta 1857:1001-1014. https://doi.org/10.1016/j.bbabio.2016.01.009

Sullivan ML (2015) Beyond brown: polyphenol oxidases as enzymes of plant specialized metabolism. Front Plant Sci 5:783. https://doi. org/10.3389/fpls.2014.00783

Tang W, Tang A-Y (2017) Transcriptional mechanisms regulating gene expression and determining cell fates in plant development. J For Res 28(5):863-880. https://doi.org/10.1007/s11676-017-0414-7

Tang X, Zhang N, Si H, Calderón-Urrea A (2017) Selection and validation of reference genes for RT-qPCR analysis in potato under 
abiotic stress. Plant Methods 13:85. https://doi.org/10.1186/s1300 7-017-0238-7

Teixeira da Silva JA, Hidvégi N, Gulyás A, Dobránszki J (2019) mRNA transcription profile of potato (Solanum tuberosum L.) in response to explant cutting. Plant Cell Tissue Organ Cult 138(1):143-152. https://doi.org/10.1007/s11240-019-01613-7

Teixeira da Silva JA, Hidvégi N, Gulyás A, Tóth B, Dobránszki J (2020) Transcriptomic response of in vitro potato (Solanum tuberosum L.) to piezoelectric ultrasound. Plant Mol Biol Rep. https://doi. org/10.1007/s11105-020-01204-3

Toro G, Pinto M (2015) Plant respiration under low oxygen. Chil J Agric Res 75(Suppl. 1):57-70. https://doi.org/10.4067/S0718-5839201500 0300007

Vandesompele J, De Preter K, Pattyn F, Poppe B, Van Roy N, De Paepe A, Speleman F (2002) Accurate normalization of real-time quantitative RT-PCR data by geometric averaging of multiple internal control genes. Genome Biol. https://doi.org/10.1186/gb-2002-3-7research0034

Wang L, Ruan Y-L (2013) Regulation of cell division and expansion by sugar and auxin signaling. Front Plant Sci 4:163. https://doi. org/10.3389/fpls.2013.00163

Wang X-C, Yang Z-R, Wang M, Meng L-Z, Jiang Y-W, Han Y-H (2014) The BRANCHING ENZYME1 gene, encoding a glycoside hydrolase family 13 protein, is required for in vitro plant regeneration in Arabidopsis. Plant Cell Tissue Organ Cult 117:279-291. https://doi. org/10.1007/s11240-014-0439-y
Widemann E, Miesch L, Lugan R, Holder E, Heinrich C, Auber Y, Miesch M, Pinot F, Heitz T (2013) The amidohydrolases IAR3 and ILL6 contribute to jasmonoyl-isoleucine hormone turnover and generate 12-hydroxyjasmonic acid upon wounding in Arabidopsis leaves. J Biol Chem 288:31701-31714. https://doi.org/10.1074/jbc. M113.499228

Williams SP, Gillaspy GE, Perera IY (2015) Biosynthesis and possible functions of inositolpyrophosphates in plants. Front Plant Sci 6:67. https://doi.org/10.3389/fpls.2015.00067

Zhang Z, Mao C-Y, Shi Z, Kou X-H (2017) The amino acid metabolic and carbohydrate metabolic pathway play important roles during salt-stress response in tomato. Front Plant Sci 8:1231. https://doi. org/10.3389/fpls.2017.01231

Zhao X-Y, Su Y-H, Cheng Z-J, Zhang X-S (2008) Cell fate switch during in vitro plant organogenesis. J Integr Plant Biol 50(7):816-824. https ://doi.org/10.1111/j.1744-7909.2008.00701.x

Zhao Y-D (2010) Auxin biosynthesis and its role in plant development. Annu Rev Plant Biol 61:49-64. https://doi.org/10.1146/annurevarplant-042809-112308

Zhuravlev YN, Omelko AM (2008) Plant morphogenesis in vitro. Russ J Plant Physiol 55(5):579-596. https://doi.org/10.1134/S102144370 8050014

Publisher's Note Springer Nature remains neutral with regard to jurisdictional claims in published maps and institutional affiliations. 